\title{
ERRATUM
}

\section{Linear Functional Differential Equations of Neutral Type Asymptotically Autonomous.}

\author{
A. F. Izé (São Paulo, Brasil)
}

There is an error in the proof of Theorem 1 and this implics that the theorem is true under a little more general condition.

Page 29, line 16. Instead of: Assume that $\pi(t)$ has a continuous derivative, $|\dot{\pi}(t)|>0, t \geqslant \sigma \geqslant 0$; should read: Assume that $\pi(t) \geqslant 0, \gamma(t) \geqslant 0$ are continuous.

Page, lines 8 to 12 should read:

$$
\begin{aligned}
& \left|\int_{\sigma}^{t} \exp [-\mu(t-\tau)-s(t, \tau)]\left\|-d_{\tau} T(t-\tau) X_{0}^{Q}\right\| G\left(\tau, W_{\tau}\right)\right|= \\
& =\mid \int_{\sigma}^{t} \exp [-s(t, x)-\beta(t-x)]\left\{d s \int_{\sigma}^{t} \exp \left[-(\mu-\beta)(x-\tau)\left\|-d_{\tau} T(x-\tau) X_{0}^{Q}\right\|_{1}\right\} G\left(x, W_{x}\right) \mid \leqslant\right. \\
& \leqslant K_{3}\left\{V \int_{\sigma}^{t} \exp [-(\mu-\beta)(t-\tau)]\left\|d_{\tau} T(t-\tau) X_{0}^{Q}\right\|\right\} \mid G\left(\xi, W_{\xi}\right) \in \operatorname{xp}[-\beta(t-\xi)] \sup _{\tau \geqslant \sigma}\left\|W_{\tau}\right\| \leqslant \\
& \leqslant K_{3}\left\{\int_{0}^{\infty}\left\|d_{2 t} T(u) X_{0}^{Q}\right\| \exp [-(\operatorname{Re} \mu-\beta) u]\right\} \pi(\xi) \exp [-\beta(t-\xi)] \sup _{\tau \geqslant \sigma}\left\|W_{\tau}\right\| \leqslant \\
& \leqslant K_{3} M \pi(\xi) \exp _{\tau \geqslant \sigma}[-\beta(t-\xi)] \sup \left\|W_{\tau}\right\| \quad \sigma \leqslant \xi \leqslant t
\end{aligned}
$$

since by Theorem 4.3, p. 120 of D. Henry, J. of Diff. Eqs., 15, (1974), $\int_{0}^{\infty}\left\|d_{u} T(u) X_{0}^{q}\right\| \exp [-(\operatorname{Re} \mu-\beta) u] \leqslant M$. When $t \rightarrow \infty$ we have two possibilities: Or $\xi=\xi(t) \rightarrow \infty$ or $\xi \rightarrow$ constant. If $\xi \rightarrow$ constant $\exp [-\beta(t-\xi)] \pi(\xi) \rightarrow 0$ for $t \rightarrow \infty$. If $\xi \rightarrow \infty$ for $t \rightarrow \infty$, as $\pi(\xi) \rightarrow 0$ for $\xi \rightarrow \infty, \pi(\xi) \exp [-\beta(t-\xi)] \rightarrow 0$ for $t \rightarrow \infty$, then we can write for simplicity $\pi(t)$ instead of $\pi(\xi) \exp [-\beta(t-\xi)]$.

Page 37, line 14. Delete $|\dot{a}(t)|>0$. 